\title{
Stress - strain behaviour of confined nano silica-based concrete
}

\author{
Kakara $S J$ Kumar $^{1}, M V$ Seshagiri $\mathrm{Rao}^{2}, V$ Srinivasa Reddy $^{3}, S$ Shrihari ${ }^{4}$ \\ ${ }^{1}$ Research Scholar of Civil Engineering, JNTUH CEH, Hyderabad. \\ ${ }^{2}$ Ex-Professor, JNTUH CEH and Professor, CVR College of Engineering, Hyderabad \\ ${ }^{3}$ Professor, Department of Civil Engineering, GRIET, Hyderabad. Email id: vempada@gmail.com \\ ${ }^{4}$ Professor, Department of Civil Engineering, VJIT, Hyderabad.
}

\begin{abstract}
In the present study, the stress-stain behaviour of confined concrete made with nanosilica (nano- $\mathrm{SiO}_{2}$ ) were taken up. The stress-strain behaviour was studied for the M30 and M50 grades nano-silica (nano- $\mathrm{SiO}_{2}$ ) concrete mixes confined with steel rebars. The confinement was given in the form of steel hoops in the cylinders, 3 hoops $(0.8 \%), 4$ hoops $(1.1 \%), 5$ hoops $(1.3 \%)$ and 6 hoops $(1.6 \%)$. The addition of nano-silica (nano- $\left.\mathrm{SiO}_{2}\right)$ along with confinement of concrete with steel hoops enhanced the compressive strength, indicating further confinement effect in the concrete. It is observed that the addition of nano-silica $\left(\right.$ nano- $\left.\mathrm{SiO}_{2}\right)$ is helpful in lower confinements only. Beyond $1.1 \%$ confinement, doesn't show any effect on compressive strengths. From the stress-strain behaviour of all types of concrete mixes, it is concluded that the ultimate load-carrying capacity and strains at peak stresses are more in nano-silica $\left(\right.$ nano- $\left.\mathrm{SiO}_{2}\right)$ concrete with steel hoops for mixes up to $1.1 \%$ confinement. The addition of nano-silica $\left(\right.$ nano- $\left.\mathrm{SiO}_{2}\right)$ to concrete has increased the ductility in both confined and unconfined states
\end{abstract}

\section{Introduction}

Concrete is a composite material consisting of many phases in micro and macro scale level. It is strong due to the heterogeneous nature acquired due to (1) interfacial transition zone between aggregate and matrix, (2) interaction between sand and paste matrix and (3) collaboration among CSH gel, large crystals of $\mathrm{Ca}(\mathrm{OH})_{2}$, unhydrated cement particles and pores formed due to high $\mathrm{w} / \mathrm{c}$ ratio. Interface between coarse aggregate, fine aggregate and cement paste is understood well with microobservational studies but the phase between the unhydrated cement grains and CSH gel was not been explored effectively especially in concretes with high w/c ratio due to availability of high amount of unhydrated cement particles. To enable a uniform distribution of nano-SiO2 in paste, colloidal nano$\mathrm{SiO}_{2}(\mathrm{CNS})$ was used instead of nano- $\mathrm{SiO}_{2}$ powder. The $\mathrm{SiO}_{2}$ content of CNS was larger than $99 \%$ by weight, and the $\mathrm{pH}$ value was 11 .

\footnotetext{
*Corresponding author: kumarjyo2003@yahoo.com
}

\section{Methodology}

In this phase of investigations, the stress-stain behaviour of nano-silica (nano- $\mathrm{SiO}_{2}$ ) concrete *confined by steel rebars were taken up. The confinement was given in the form of steel hoops in the cylinders, 3 hoops $(0.8 \%), 4$ hoops $(1.1 \%), 5$ hoops $(1.3 \%)$ and 6 hoops (1.6\%) as shown in Fig 1. The tests were carried out on the standard cylindrical specimens of diameter $150 \mathrm{~mm}$ and height $300 \mathrm{~mm}$. After casting, the cylinders were capped with cement mortar and cured for a period of 28 days in curing tanks. The specimens were then taken out and made surface dry. The samples were placed in a microprocessor strain controlled universal testing machine of $1000 \mathrm{kN}$ capacity and tested under uniaxial compression as per IS 516:1959. The stressstrain behaviour as obtained was plotted. 


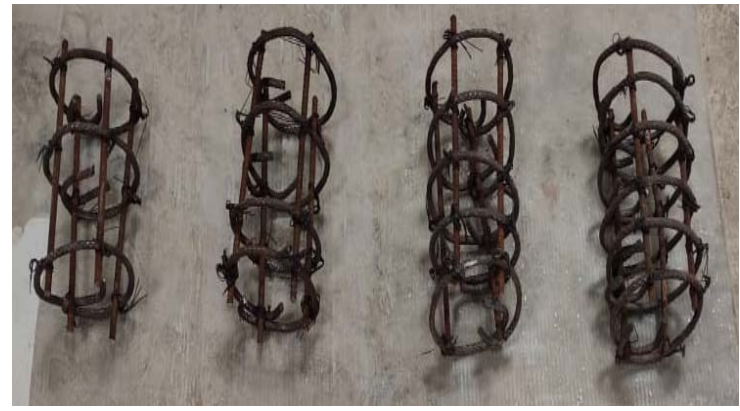

Fig.1. Cylinders with different Confinements

Table 1. Percentage of confinement by volume

\begin{tabular}{|c|c|c|}
\hline & & Volume of confinement percentage \\
\hline C0 & Confinement 0 hoops & 0.00 \\
\hline C3 & Confinement 3 hoops & 0.80 \\
\hline C4 & Confinement 4 hoops & 1.1 \\
\hline C5 & Confinement 5 hoops & 1.3 \\
\hline C6 & Confinement 6 hoops & 1.6 \\
\hline
\end{tabular}

Table 2. Stress-strain values of M30 normal concrete confined with steel bars

\begin{tabular}{|c|c|c|c|c|c|c|c|c|c|}
\hline \multicolumn{2}{|c|}{ M30SCC -C0 } & \multicolumn{2}{|c|}{ M30SCC -C3 } & \multicolumn{2}{c|}{ M30SCC -C4 } & \multicolumn{2}{c|}{ M30SCC -C5 } & \multicolumn{2}{c|}{ M30SCC -C6 } \\
\hline Strain & Stress & Strain & Stress & Strain & Stress & Strain & Stress & Strain & Stress \\
\hline 0.0000 & 0.00 & 0.0000 & 0.00 & 0.0000 & 0.00 & 0.0000 & 0.00 & 0.0000 & 0.00 \\
\hline 0.0003 & 5.24 & 0.0000 & 1.17 & 0.0000 & 0.39 & 0.0000 & 2.16 & 0.0000 & 0.86 \\
\hline 0.0004 & 9.43 & 0.0002 & 7.39 & 0.0003 & 5.50 & 0.0002 & 9.05 & 0.0000 & 0.00 \\
\hline 0.0007 & 13.97 & 0.0004 & 12.45 & 0.0007 & 14.15 & 0.0005 & 15.95 & 0.0001 & 0.87 \\
\hline 0.0009 & 17.82 & 0.0007 & 18.68 & 0.0011 & 21.22 & 0.0008 & 22.84 & 0.0003 & 5.63 \\
\hline 0.0011 & 23.41 & 0.0010 & 24.53 & 0.0015 & 30.66 & 0.0011 & 33.62 & 0.0006 & 13.41 \\
\hline 0.0013 & 25.15 & 0.0015 & 34.27 & 0.0017 & 39.30 & 0.0014 & 40.95 & 0.0009 & 24.21 \\
\hline 0.0017 & 32.49 & 0.0018 & 42.45 & 0.0020 & 46.38 & 0.0018 & 49.14 & 0.0012 & 32.86 \\
\hline 0.0021 & 39.13 & 0.0022 & 50.63 & 0.0025 & 53.06 & 0.0021 & 56.90 & 0.0015 & 42.79 \\
\hline 0.0023 & 43.67 & 0.0026 & 59.20 & 0.0028 & 57.38 & 0.0024 & 60.34 & 0.0019 & 49.29 \\
\hline 0.0026 & 48.91 & 0.0030 & 65.05 & 0.0031 & 64.85 & 0.0027 & 67.24 & 0.0022 & 56.65 \\
\hline 0.0027 & 52.05 & 0.0031 & 67.39 & 0.0036 & 71.92 & 0.0030 & 75.86 & 0.0027 & 64.88 \\
\hline 0.0029 & 56.94 & 0.0035 & 71.30 & 0.0039 & 74.28 & 0.0033 & 78.45 & 0.0030 & 72.24 \\
\hline 0.0032 & 62.18 & 0.0038 & 74.04 & 0.0042 & 77.42 & 0.0036 & 80.60 & 0.0033 & 77.86 \\
\hline 0.0035 & 64.28 & 0.0041 & 78.34 & 0.0045 & 80.57 & 0.0040 & 81.47 & 0.0036 & 81.77 \\
\hline 0.0037 & 64.98 & 0.0042 & 81.46 & 0.0047 & 83.32 & 0.0043 & 83.19 & 0.0039 & 84.81 \\
\hline 0.0041 & 70.22 & 0.0045 & 81.48 & 0.0050 & 84.50 & 0.0044 & 83.62 & 0.0043 & 87.86 \\
\hline 0.0043 & 66.38 & 0.0048 & 80.73 & 0.0055 & 84.89 & 0.0049 & 87.07 & 0.0048 & 88.76 \\
\hline 0.0044 & 62.53 & 0.0051 & 77.65 & 0.0058 & 77.82 & 0.0052 & 87.07 & 0.0051 & 90.52 \\
\hline 0.0046 & 55.90 & 0.0051 & 72.62 & 0.0059 & 71.53 & 0.0055 & 87.07 & 0.0055 & 88.39 \\
\hline 0.0047 & 49.26 & 0.0052 & 67.19 & 0.0060 & 64.06 & 0.0058 & 81.90 & 0.0058 & 84.54 \\
\hline & & 0.0053 & 63.70 & & & 0.0059 & 75.43 & 0.0062 & 76.82 \\
\hline & & & & & & 0.0060 & 67.24 & & \\
\hline
\end{tabular}




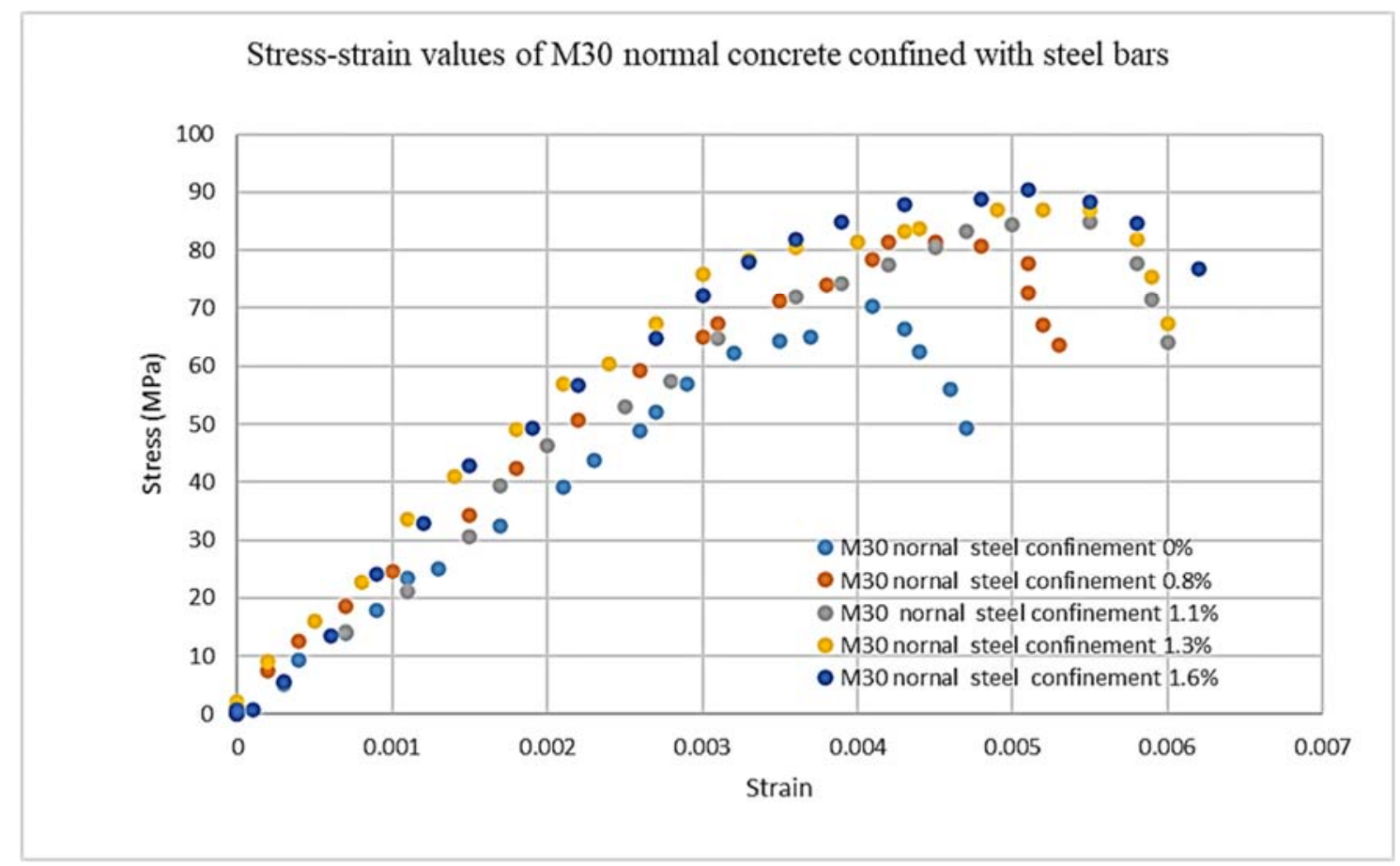

Fig.1. Stress-strain curves of M30 normal concrete confined with steel bars

Table3. Stress-strain values of M30 nano silica concrete confined with steel bars

\begin{tabular}{|c|c|c|c|c|c|c|c|c|c|}
\hline \multicolumn{2}{|c|}{ M30SCC NS-C0 } & \multicolumn{2}{c|}{ M30SCC NS-C3 } & \multicolumn{2}{c|}{ M30SCC NS-C4 } & \multicolumn{2}{c|}{ M30SCC NS-C5 } & \multicolumn{2}{c|}{ M30SCC NS-C6 } \\
\hline Strain & Stress & Strain & Stress & Strain & Stress & Strain & Stress & Strain & Stress \\
\hline 0.0000 & 0.00 & 0.0000 & 0.00 & 0.0000 & 0.00 & 0.0000 & 0.00 & 0.0000 & 0.00 \\
\hline 0.0002 & 8.66 & 0.0003 & 7.27 & 0.0004 & 6.90 & 0.0004 & 7.79 & 0.0000 & 0.87 \\
\hline 0.0004 & 13.85 & 0.0006 & 16.28 & 0.0006 & 19.47 & 0.0005 & 14.29 & 0.0003 & 7.42 \\
\hline 0.0007 & 19.74 & 0.0010 & 24.94 & 0.0011 & 27.63 & 0.0006 & 21.21 & 0.0005 & 13.52 \\
\hline 0.0009 & 25.97 & 0.0013 & 32.55 & 0.0017 & 39.71 & 0.0009 & 26.41 & 0.0007 & 20.49 \\
\hline 0.0011 & 29.44 & 0.0015 & 37.06 & 0.0022 & 50.93 & 0.0011 & 31.60 & 0.0010 & 29.21 \\
\hline 0.0014 & 34.63 & 0.0018 & 40.52 & 0.0027 & 62.14 & 0.0012 & 36.80 & 0.0011 & 37.05 \\
\hline 0.0017 & 41.90 & 0.0020 & 45.71 & 0.0032 & 68.15 & 0.0015 & 41.99 & 0.0013 & 41.42 \\
\hline 0.0021 & 46.75 & 0.0022 & 49.87 & 0.0037 & 74.14 & 0.0018 & 47.19 & 0.0016 & 45.79 \\
\hline 0.0023 & 52.29 & 0.0024 & 54.72 & 0.0040 & 76.71 & 0.0020 & 51.95 & 0.0019 & 54.51 \\
\hline 0.0025 & 54.72 & 0.0028 & 60.26 & 0.0043 & 80.13 & 0.0022 & 57.58 & 0.0021 & 58.87 \\
\hline 0.0027 & 59.57 & 0.0032 & 64.42 & 0.0044 & 82.28 & 0.0026 & 62.34 & 0.0023 & 62.80 \\
\hline 0.0031 & 64.42 & 0.0035 & 67.88 & 0.0047 & 84.41 & 0.0028 & 67.53 & 0.0026 & 68.92 \\
\hline 0.0033 & 64.76 & 0.0039 & 73.42 & 0.0049 & 83.93 & 0.0032 & 71.86 & 0.0029 & 72.85 \\
\hline 0.0035 & 68.57 & 0.0043 & 73.42 & 0.0052 & 79.54 & 0.0035 & 78.35 & 0.0031 & 77.66 \\
\hline 0.0038 & 74.46 & 0.0046 & 67.19 & 0.0055 & 72.97 & 0.0038 & 80.95 & 0.0034 & 82.03 \\
\hline 0.0043 & 69.61 & 0.0049 & 60.95 & 0.0058 & 65.53 & 0.0041 & 83.98 & 0.0041 & 86.87 \\
\hline 0.0043 & 65.11 & 0.0050 & 55.76 & & & 0.0045 & 85.71 & 0.0043 & 88.63 \\
\hline 0.0046 & 59.22 & 0.0051 & 49.52 & & & 0.0048 & 87.45 & 0.0046 & 90.82 \\
\hline 0.0047 & 54.03 & & & & & 0.0050 & 86.15 & 0.0050 & 93.90 \\
\hline 0.0050 & 51.95 & & & & & 0.0052 & 84.85 & 0.0053 & 95.66 \\
\hline & & & & & & 0.0055 & 83.98 & 0.0057 & 96.57 \\
\hline & & & & & & 0.0057 & 79.22 & 0.0059 & 90.50 \\
\hline & & & & & & 0.0059 & 72.29 & 0.0060 & 86.16 \\
\hline & & & & & & & & 0.0060 & 78.77 \\
\hline
\end{tabular}




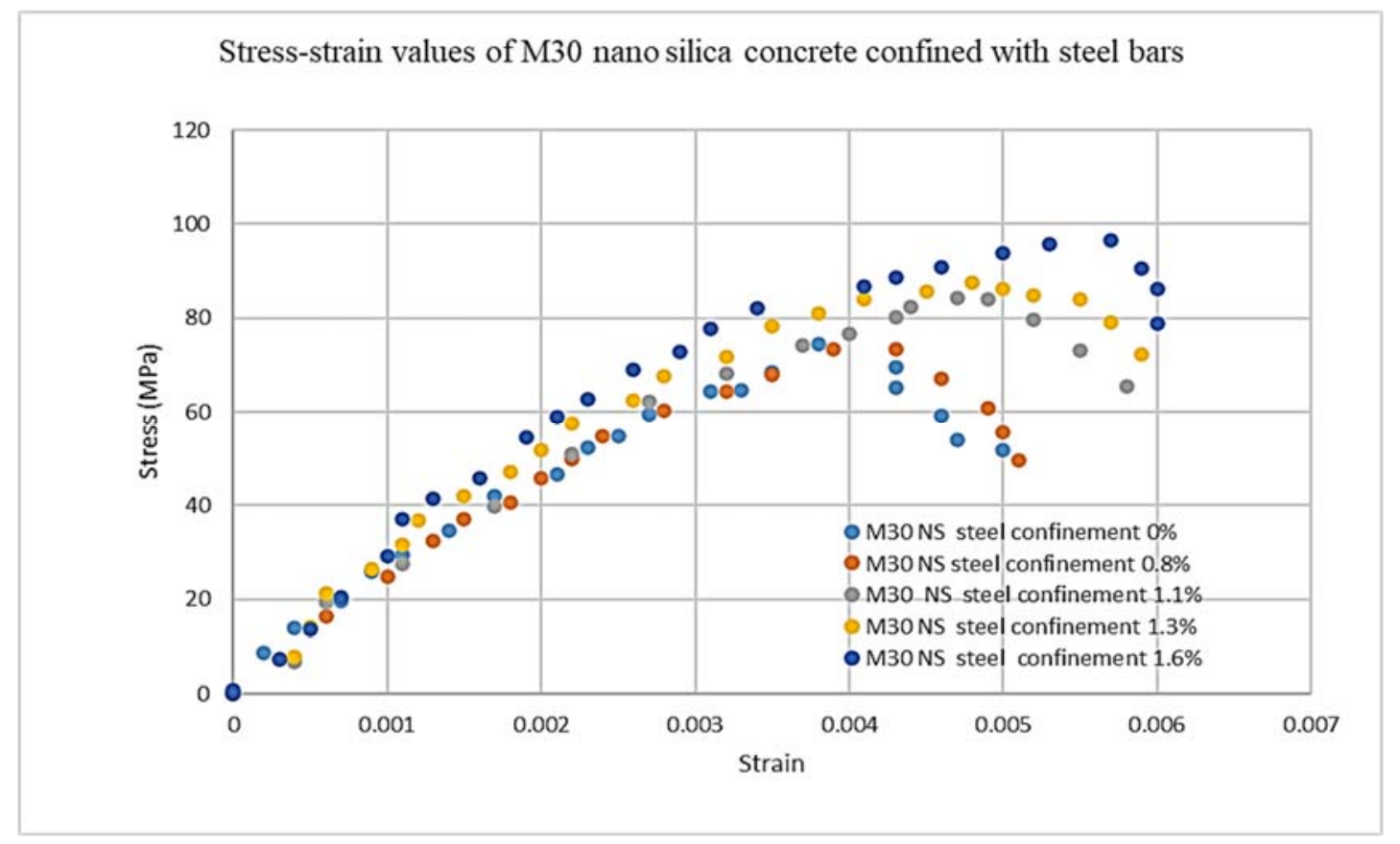

Fig.2. Stress-strain curves of M30 nano silica concrete confined with steel bars

Table 4. Stress-strain values of M50 normal concrete confined with steel bars

\begin{tabular}{|c|c|c|c|c|c|c|c|c|c|}
\hline \multicolumn{2}{|c|}{ M50SCC -C0 } & \multicolumn{2}{|c|}{ M50SCC -C3 } & \multicolumn{2}{|c|}{ M50SCC -C4 } & \multicolumn{2}{|c|}{ M50SCC -C5 } & \multicolumn{2}{|c|}{ M50SCC -C6 } \\
\hline Strain & Stress & Strain & Stress & Strain & Stress & Strain & Stress & Strain & Stress \\
\hline 0.0000 & 0.00 & 0.0000 & 0.00 & 0.0000 & 0.00 & 0.0000 & 0.00 & 0.0000 & 0.00 \\
\hline 0.0003 & 6.99 & 0.0002 & 4.37 & 0.0003 & 6.01 & 0.0000 & 0.43 & 0.0002 & 5.74 \\
\hline 0.0005 & 10.13 & 0.0005 & 11.35 & 0.0005 & 12.03 & 0.0003 & 6.44 & 0.0004 & 14.61 \\
\hline 0.0006 & 15.02 & 0.0007 & 17.89 & 0.0007 & 18.48 & 0.0005 & 13.75 & 0.0007 & 20.87 \\
\hline 0.0009 & 20.26 & 0.0010 & 23.56 & 0.0009 & 24.92 & 0.0007 & 19.77 & 0.0008 & 27.65 \\
\hline 0.0011 & 24.80 & 0.0012 & 31.41 & 0.0012 & 30.50 & 0.0010 & 27.08 & 0.0011 & 35.48 \\
\hline 0.0014 & 30.74 & 0.0014 & 36.21 & 0.0014 & 35.66 & 0.0012 & 32.23 & 0.0013 & 44.87 \\
\hline 0.0016 & 34.59 & 0.0017 & 40.58 & 0.0016 & 43.83 & 0.0014 & 38.25 & 0.0016 & 52.70 \\
\hline 0.0018 & 39.13 & 0.0019 & 47.55 & 0.0018 & 48.55 & 0.0016 & 45.12 & 0.0019 & 58.43 \\
\hline 0.0021 & 44.37 & 0.0021 & 51.05 & 0.0021 & 55.00 & 0.0019 & 48.98 & 0.0022 & 63.65 \\
\hline 0.0024 & 47.51 & 0.0024 & 55.42 & 0.0023 & 58.86 & 0.0022 & 56.28 & 0.0026 & 69.39 \\
\hline 0.0026 & 52.05 & 0.0026 & 60.23 & 0.0026 & 64.01 & 0.0024 & 60.14 & 0.0029 & 73.04 \\
\hline 0.0029 & 57.29 & 0.0029 & 65.90 & 0.0028 & 67.43 & 0.0027 & 64.86 & 0.0031 & 77.74 \\
\hline 0.0031 & 60.09 & 0.0031 & 68.96 & 0.0031 & 73.01 & 0.0030 & 67.42 & 0.0035 & 82.96 \\
\hline 0.0033 & 62.18 & 0.0034 & 72.90 & 0.0035 & 75.57 & 0.0032 & 70.85 & 0.0038 & 85.57 \\
\hline 0.0037 & 66.38 & 0.0036 & 75.53 & 0.0038 & 78.99 & 0.0035 & 74.28 & 0.0040 & 88.70 \\
\hline 0.0039 & 68.47 & 0.0040 & 80.34 & 0.0040 & 81.13 & 0.0038 & 77.70 & 0.0043 & 91.83 \\
\hline 0.0045 & 76.16 & 0.0047 & 88.22 & 0.0043 & 83.69 & 0.0040 & 80.70 & 0.0047 & 93.91 \\
\hline 0.0049 & 75.46 & 0.0052 & 86.97 & 0.0045 & 86.26 & 0.0041 & 83.71 & 0.0051 & 93.91 \\
\hline 0.0052 & 71.62 & 0.0056 & 81.78 & 0.0049 & 89.24 & 0.0046 & 84.96 & 0.0053 & 95.48 \\
\hline 0.0055 & 66.03 & 0.0059 & 68.77 & 0.0051 & 89.66 & 0.0049 & 86.23 & 0.0054 & 96.00 \\
\hline 0.0058 & 62.18 & & & 0.0054 & 89.20 & 0.0051 & 88.80 & 0.0058 & 95.48 \\
\hline 0.0059 & 56.24 & & & 0.0057 & 86.59 & 0.0056 & 87.90 & 0.0060 & 89.74 \\
\hline 0.0061 & 51.70 & & & 0.0059 & 83.55 & 0.0057 & 83.57 & 0.0061 & 84.00 \\
\hline & & & & 0.0060 & 77.51 & 0.0059 & 78.39 & 0.0063 & 75.13 \\
\hline & & & & & & 0.0060 & 74.06 & & \\
\hline & & & & & & 0.0061 & 67.16 & & \\
\hline
\end{tabular}






Fig.3. Stress-strain curves of M30 normal concrete confined with steel bars

Table 5. Stress-strain values of M50 nano silica concrete confined with steel bars

\begin{tabular}{|c|c|c|c|c|c|c|c|c|c|}
\hline \multicolumn{2}{|c|}{ M50SCC N S 0} & \multicolumn{2}{c|}{ M50SCC NS-C3 } & \multicolumn{2}{c|}{ M50SCC NS-C4 } & \multicolumn{2}{c|}{ M50SCC NS-C5 } & \multicolumn{2}{c|}{ M50SCC NS-C6 } \\
\hline Strain & Stress & Strain & Stress & Strain & Stress & Strain & Stress & Strain & Stress \\
\hline 0.0000 & 0.00 & 0.0000 & 0.00 & 0.0000 & 0.00 & 0.0000 & 0.00 & 0.0000 & 0.00 \\
\hline 0.0001 & 0.70 & 0.0001 & 3.17 & 0.0002 & 5.64 & 0.0002 & 6.89 & 0.0002 & 7.29 \\
\hline 0.0002 & 5.56 & 0.0003 & 9.50 & 0.0004 & 13.44 & 0.0004 & 15.94 & 0.0005 & 15.08 \\
\hline 0.0005 & 11.81 & 0.0006 & 15.22 & 0.0008 & 18.63 & 0.0006 & 22.41 & 0.0007 & 22.37 \\
\hline 0.0007 & 18.40 & 0.0008 & 22.42 & 0.0009 & 26.45 & 0.0009 & 29.29 & 0.0010 & 29.65 \\
\hline 0.0010 & 22.23 & 0.0010 & 27.59 & 0.0011 & 32.95 & 0.0011 & 33.57 & 0.0011 & 37.46 \\
\hline 0.0012 & 26.75 & 0.0013 & 32.44 & 0.0014 & 38.14 & 0.0014 & 38.73 & 0.0014 & 43.70 \\
\hline 0.0014 & 33.34 & 0.0015 & 39.43 & 0.0016 & 45.51 & 0.0017 & 45.16 & 0.0017 & 49.93 \\
\hline 0.0017 & 38.21 & 0.0017 & 44.68 & 0.0018 & 51.15 & 0.0019 & 50.75 & 0.0021 & 59.29 \\
\hline 0.0019 & 42.04 & 0.0020 & 49.62 & 0.0021 & 57.21 & 0.0023 & 58.06 & 0.0024 & 69.17 \\
\hline 0.0022 & 47.25 & 0.0023 & 55.26 & 0.0024 & 63.27 & 0.0025 & 62.79 & 0.0028 & 73.84 \\
\hline 0.0024 & 49.69 & 0.0026 & 59.29 & 0.0028 & 68.89 & 0.0027 & 67.94 & 0.0031 & 79.02 \\
\hline 0.0026 & 53.86 & 0.0029 & 63.32 & 0.0031 & 72.77 & 0.0029 & 73.09 & 0.0035 & 87.33 \\
\hline 0.0029 & 57.69 & 0.0032 & 67.39 & 0.0035 & 77.09 & 0.0033 & 78.66 & 0.0039 & 90.43 \\
\hline 0.0032 & 61.17 & 0.0035 & 71.73 & 0.0038 & 82.29 & 0.0036 & 82.94 & 0.0044 & 93.51 \\
\hline 0.0034 & 64.30 & 0.0038 & 74.58 & 0.0041 & 84.86 & 0.0039 & 86.34 & 0.0049 & 93.99 \\
\hline 0.0037 & 68.83 & 0.0041 & 77.05 & 0.0044 & 85.27 & 0.0043 & 87.58 & 0.0052 & 96.05 \\
\hline 0.0040 & 70.92 & 0.0043 & 78.96 & 0.0047 & 86.99 & 0.0049 & 88.79 & 0.0056 & 98.10 \\
\hline 0.0041 & 73.70 & 0.0046 & 81.85 & 0.0050 & 90.00 & 0.0051 & 89.18 & 0.0060 & 95.98 \\
\hline 0.0045 & 75.46 & 0.0049 & 82.50 & 0.0054 & 89.54 & 0.0053 & 90.88 & 0.0064 & 89.68 \\
\hline 0.0048 & 76.17 & 0.0052 & 80.67 & 0.0056 & 85.17 & 0.0058 & 89.49 & 0.0067 & 83.92 \\
\hline 0.0053 & 74.48 & 0.0055 & 77.65 & 0.0058 & 80.81 & 0.0061 & 86.42 & 0.0070 & 77.11 \\
\hline 0.0055 & 69.65 & 0.0058 & 72.39 & 0.0060 & 75.14 & 0.0064 & 79.01 & & \\
\hline 0.0057 & 64.12 & 0.0029 & 32.06 & & & 0.0065 & 72.49 & & \\
\hline 0.0060 & 57.90 & 0.0030 & 28.95 & & & 0.0066 & 65.98 & & \\
\hline
\end{tabular}






Fig.4. Stress-strain curves of M30 normal concrete confined with steel bars

\section{Conclusions}

1. The addition of nano silica along with confinement with steel hoops enhanced the compressive strength, indicating further confinement effect in the concrete.

2. It is observed that the addition of nano silica is helpful in lower confinements only. Beyond $1.1 \%$ confinement, doesn't show any effect on compressive strengths.

3. From the stress-strain behaviour of normal and nano silica-based concrete in M30 and M50 grade concretes, it is concluded that the ultimate load-carrying capacity and strains at peak stresses are more in nano silica mixes up to $1.1 \%$ confinement.

4. The addition of nano silica to concrete has increased the ductility in both confined and unconfined states.

\section{References}

1. T.Srinivas and M. Abinay Raj, Int. J. of Eng.and Adv. Tech. (IJEAT), ISSN: 2249 - 8958, Volume-8 Issue-6 (2019)

2. T.srinivas and P. Manoj Anand, Int. J. of Innov. Tech. and Explor. Eng.g (IJITEE), ISSN: 22783075, Volume-8 Issue-12 (2019)

3. T.Srinivas and G. Sukesh Reddy, Int. J. of Eng.and Adv. Tech. (IJEAT), ISSN: $2249-$ 8958, Volume-9 Issue-1 (2019)

4. T.Srinivas and R. N. Koushik, Int. J. of Innov. Tech. and Explor. Eng.g (IJITEE), ISSN: 22783075, Volume-8 Issue-12 (2019), PP 112-117.
5. K. Sai Gopi, Dr. T. Srinivas and S. P. Raju V, E3S Web of Conferences ICMED 184, 01084GRIET, 28-29 February, https://doi.org/10.1051/e3sconf/202018401108 4(2020)

6. Jagannadha Kumar, M.V., Jagannadha Rao, K., Dean Kumar, B., Srinivasa Reddy, V., Int. J. of Civil Eng. and Tech., 9(7), pp. 1133-1141 (2018)

7. Ganta, J.K., Seshagiri Rao, M.V., Mousavi, S.S., Srinivasa Reddy, V., Bhojaraju, C., Structures 28, pp. 956-972 (2020)

8. Srinivas Rao J, S K Tummala, Kuthuri N R, Indonesia Journal of Electrical Engg. \& Computer Science, 21 (723), 2020

9. Naidu, K.S.S.T., Rao, M.V.S., Reddy, V.S., Int. J. of Innov. Tech. and Explor. Eng.g (IJITEE), 8(9 Special Issue 2), pp. 641-642 (2019)

10. Chandana Priya, C., Seshagiri Rao, M.V., Srinivasa Reddy, V., Int. J. of Civil Eng. and Tech., 9(11), pp. 2218-2225 (2018)

11. K. Satyanarayana, M.V. Kumar, and N. Sateesh, Materials Today: Proceedings, 5(2), 5343 (2018).

12. Satya Sai Trimurty Naidu, K., Seshagiri Rao, M.V., Srinivasa Reddy, V., Int. J. of Civil Eng. and Tech., 9(11), pp. 2383-2393 (2018)

13. Supriya, Y., Srinivasa Reddy, V., Seshagiri Rao, M.V., Shrihari, S., Int. J. of Rec. Tech. and Engi., 8(3), pp. 5381-5385 (2019)

14. B. J. Varghese, P. B. Bobba and M. Kavitha, 2016 IEEE 7th Power India International Conference (PIICON), 2016, pp. 1-6

15. Kotkunde, N., Krishna, G., Shenoy, S.K., Gupta, A.K., Singh, S.K. International Journal of Material Forming, 10 (2), pp. 255-266 (2017) 
16. Govardhan, D., Kumar, A.C.S., Murti, K.G.K., Madhusudhan Reddy, G. Materials and Design, 36, pp. 206-214. (2012)

17. Kumar, P., Singhal, A., Mehta, S., Mittal, A. Journal of Real-Time Image Processing, 11 (1), pp. 93-109. (2016)

18. Raghunadha Reddy, T., Vishnu Vardhan, B., Vijayapal Reddy, P. International Journal of Applied Engineering Research, 11 (5), pp. 30923102 (2016)
19. Hussaini, S.M., Krishna, G., Gupta, A.K., Singh, S.K. Journal of Manufacturing Processes, 18, pp. 151-158 (2015)

20. Karthik Rao, R., Bobba, P.B., Suresh Kumar, T., Kosaraju, S. Materials Today: Proceedings, 26, pp. 3085-3089, 2019 